EUROPEAN ORGANIZATION FOR NUCLEAR RESEARCH

CERN-EP/2003-029

03 April 2003

\title{
Recent improvements of ISOLTRAP: Absolute mass measurements of exotic nuclides at $10^{-8}$ precision
}

\author{
A. Kellerbauer ${ }^{1)}$ \\ for the ISOLTRAP Collaboration
}

\begin{abstract}
In the past three years, the sensitivity and the performance of the Penning trap mass spectrometer ISOLTRAP have been enhanced significantly. These improvements, which range from technical developments to systematic studies of the various factors contributing to the uncertainty of the final mass result, now allow mass measurements of short-lived radionuclides with half-lives of less than $100 \mathrm{~ms}$ and with a precision of better than $10^{-8}$. Using a newly developed carbon cluster ion source, ISOLTRAP can perform absolute mass measurements relative to the microscopic mass standard ${ }^{12} \mathrm{C}$. These developments are reviewed as pertaining to the extension of ISOLTRAP mass measurements to higher precision and shorter half-lives and to molecular mass measurements.
\end{abstract}

PACS: 07.75. $+\mathrm{h}$ (Mass spectrometers and related techniques), 21.10.Dr (Binding energies and masses), 32.10.Bi (Atomic masses, mass spectra, abundances, and isotopes), 36.40.Wa (Charged clusters).

(Accepted for publication in International Journal of Mass Spectrometry)

1) CERN Division EP, 1211 Genève 23, Switzerland 



\section{Introduction}

The mass is one of the most fundamental properties of an atom and is of great importance both in chemistry and physics. High-precision atomic mass measurements convey direct information on nuclear binding energies and are therefore of interest for a large number of fields of physics [1]: By determining differences in binding energies along isobaric or isotonic chains, they allow the observation of nuclear-structure effects such as pairing, shell closures and deformation [2]. The masses of exotic nuclides, along with their half-lives, are crucial parameters for calculations of nucleosynthesis processes in astrophysics [3]. Furthermore, highly precise measurements of $\beta$ decay energies are required for a nuclear-physics contribution to fundamental studies such as a check of the conservedvector-current (CVC) hypothesis and Standard-Model tests such as a verification of the unitarity of the Cabibbo-Kobayashi-Maskawa (CKM) matrix [4]. The required precision of the measurements ranges from $10^{-7}$ for shell-model calculations to $10^{-8}$ and better for Standard-Model tests. In addition to these precision requirements, the radionuclides of interest are often produced in only minute quantities of a few tens or hundreds ions per second and it is therefore crucial to reach the highest possible efficiency throughout the measurement cycle in order not to add losses to the radioactive decay of the observed nuclides.

The currently highest precisions of direct mass measurements are reached with Penning trap mass spectrometers using single-ion detection: Using highly charged ions and/or very long observation times, both SMILETRAP in Stockholm [5] and the Penning trap setup at the University of Mainz [6] have achieved uncertainties of only a few parts in $10^{-10}$. Thanks to a novel technique in which two different ions (atomic or molecular) with the same mass number are trapped simultaneously, a precision of close to $10^{-11}$ is obtained with the Penning trap at MIT [7]. For mass measurements on radioactive nuclides, one of the world's most prominent instruments today, both in terms of the final mass uncertainty reached and in terms of its sensitivity and the number of measurements performed, is the Penning trap mass spectrometer ISOLTRAP [8] installed at the on-line isotope separator facility ISOLDE [9] at CERN (Geneva, Switzerland).

ISOLTRAP looks back on a highly successful physics program since its installation at the original ISOLDE facility in 1992. It has been used to measure the masses of more than 200 radioactive nuclides throughout the entire chart of the nuclides $[10,11]$. Before the improvements discussed here, its overall relative mass uncertainty (or the limit of its accuracy) was estimated to be $1 \cdot 10^{-7}$, and the efficiency was typically a few times $10^{-7}[8,12]$. While this was sufficient for most nuclear-physics applications, the fundamental studies mentioned above have made it desirable to improve that precision by at least an order of magnitude and the sensitivity by more than three orders of magnitude.

\section{$2 \quad$ Principle and setup}

A mass measurement with ISOLTRAP is based on the determination of the cyclotron frequency $\nu_{\mathrm{c}}$ of an ion with mass $m$ and charge $q$ in a magnetic field of magnitude $B$ :

$$
\nu_{\mathrm{c}}=\frac{1}{2 \pi} \frac{q}{m} B
$$

The magnitude of the magnetic field is inferred from the cyclotron frequency measurement of an ion whose mass is already known to high precision, a so-called reference ion. Typically, the stable nuclides used as reference masses are ${ }^{39} \mathrm{~K},{ }^{85} \mathrm{Rb}$, and ${ }^{133} \mathrm{Cs}$. The latter two are known to relative precisions at the $10^{-10}$ level [13]. 


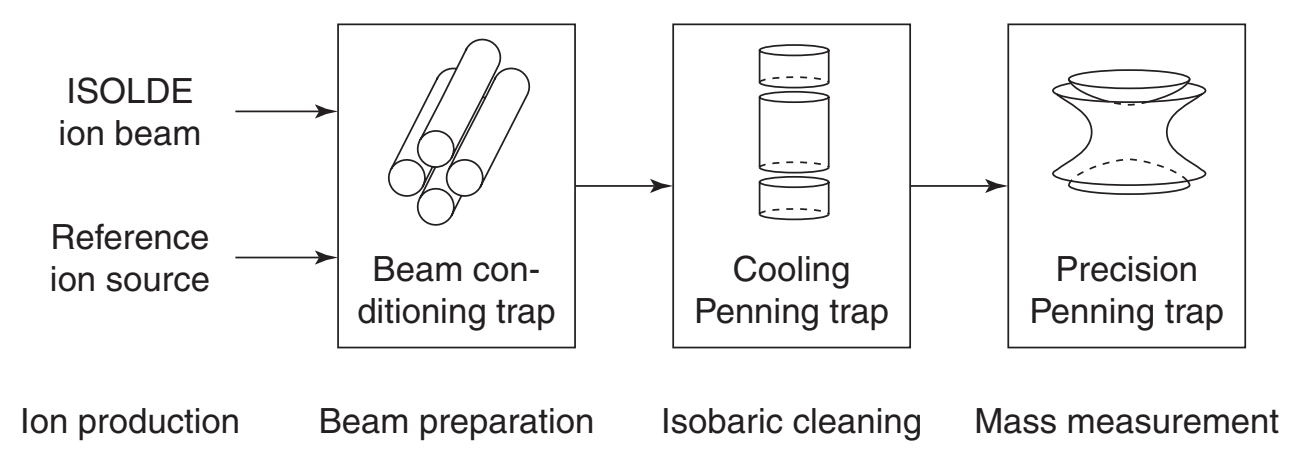

Figure 1: Sketch of the main components of the ISOLTRAP mass spectrometer and their functions.

The structure of the ISOLTRAP apparatus is organized according to the principle of separation of function [8]. Figure 1 shows a functional diagram with the main components of the setup. It consists of three separate traps that perform specific specialized tasks: (i) the beam conditioning trap in which the 60-keV ISOLDE beam is decelerated, cooled, and bunched to adapt the beam to the requirements of ISOLTRAP with respect to its time structure and emittance; (ii) the cooling Penning trap [14], in which contaminant ions are removed by use of a mass-selective cooling scheme [15]; and (iii) the precision Penning trap, in which the actual mass measurement is carried out. A stable alkali reference ion source located upstream of the beam conditioning trap allows the testing and preparation of the complete setup before radioactive experiments. Furthermore, the nuclides produced in this ion source are used as reference ions for mass measurements.

In the field configuration of a Penning trap - a homogeneous magnetic field superimposed onto a quadrupolar electric field - an ion performs a superposition of three harmonic motions: an axial mode with frequency $\nu_{z}$ and two radial modes, a low-frequency magnetron motion with frequency $\nu_{-}$and a high-frequency modified cyclotron motion with frequency $\nu_{+}[16]$. The two radial modes can be coupled by an excitation with an azimuthal quadrupolar electric RF field with a frequency near the sum of the frequencies of the two radial modes. This sum frequency is in fact exactly the true cyclotron frequency of an ion in a purely magnetic field:

$$
\nu_{+}+\nu_{-}=\nu_{\mathrm{c}}
$$

As a result of this coupling, the two motions are continuously converted into each other, in a manner analogous to the way kinetic energy is transferred back and forth in a system of coupled pendulums.

This behavior is exploited in the time-of-flight (TOF) cyclotron resonance detection technique [17]. Initially, the ions of interest are essentially at rest in the precision Penning trap. First, their magnetron motion is resonantly excited by an azimuthal dipolar electric AC field with frequency $\nu_{\mathrm{D}}=\nu_{-}$. The ions are then exposed to an azimuthal quadrupolar electric RF field whose frequency $\nu_{\mathrm{Q}}$ is scanned around the (expected) cyclotron frequency. When the excitation is resonant, the ions' magnetron motion is converted into higher-frequency modified cyclotron motion. After this observation time, the ion cloud is electrostatically accelerated out of the trap towards a micro-channel plate (MCP) detector. In the inhomogeneous-magnetic-field region along the trajectory between the trap and the detector, the magnetic-field gradient exerts a linear force on the magnetic moment of each ion, accelerating it towards the detector. This force is proportional to the 


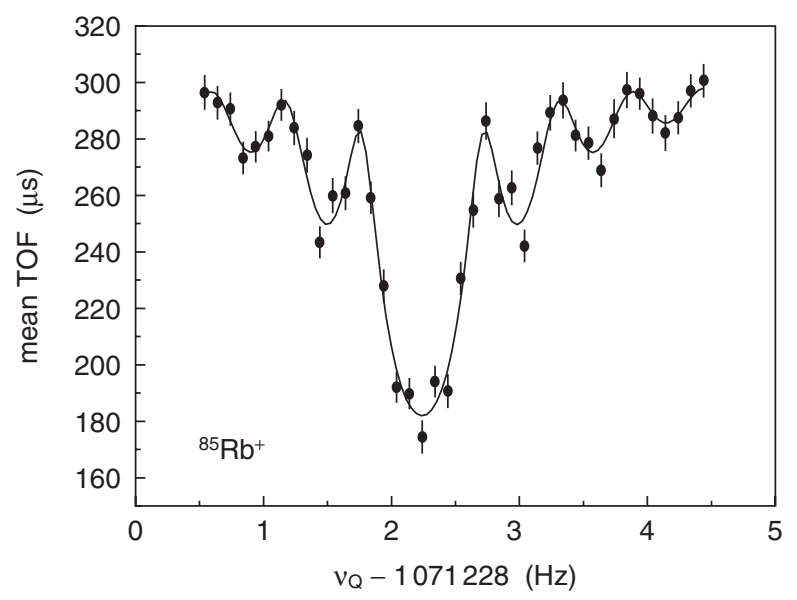

Figure 2: Time-of-flight resonance curve of ${ }^{85} \mathrm{Rb}^{+}$at an excitation time of $T_{\mathrm{Q}}=1.8 \mathrm{~s}$. The solid line is a fit of the theoretically expected line shape [17] to the data points.

magnetic moment and thus proportional to the initial radial energy. The ions whose radial motion has been converted into higher-energy modified cyclotron motion therefore reach the detector after a reduced time of flight.

A typical TOF cyclotron resonance for ${ }^{85} \mathrm{Rb}^{+}$is shown in Fig. 2. The solid line is a fit of the theoretically expected resonance line shape [17] to the data points.

\section{$3 \quad$ Resolution and precision}

Fundamentally, the primary prerequisite for the precision of an instrument is its resolving power, i.e. the line width of a resonance obtained with it [1]. According to the Rayleigh criterion, two peaks are resolved if the separation of two centroids is at least as large as the distance from either of the centroids to its first minimum. For measurements using the TOF resonance detection technique, the frequency resolving power $R$ according to this criterion is

$$
R=\nu_{\mathrm{c}} T_{\mathrm{Q}},
$$

where $T_{\mathrm{Q}}$ is the duration of the quadrupolar excitation, i.e. the observation time in the precision Penning trap [16].

The ability to reduce the precision beyond the line width of the centroid peak depends on the understanding of the resonance line shape and therefore the theoretical description, as well as the statistical uncertainty of the data points that compose the measurement. Typically, a centroid can be "split" by a factor 1 to 10. Owing to the excellent understanding of the underlying excitation and conversion process [17], a splitting factor of up to 100 can be reached with ISOLTRAP measurements.

Based on these realizations, the following relation for the statistical uncertainty $s\left(\nu_{\mathrm{c}}\right)$ of the cyclotron frequency has been proposed [10] and verified [18]:

$$
s\left(\nu_{\mathrm{c}}\right) \propto \frac{1}{\sqrt{N_{\text {tot }}} T_{\mathrm{Q}}},
$$

where $N_{\text {tot }}$ is the total number of ions recorded in a single resonance and $T_{\mathrm{Q}}$ is the duration of the quadrupolar radiofrequency excitation in the precision trap. In a radioactive-beam experiment, one has to take into account the fact that the number of ions of a radionuclide being confined in the precision trap decreases exponentially due to radioactive decay. 
Plugging this decrease of the form $N_{\text {tot }}=N_{\text {tot }}(0) \exp (-t / \tau)$ into Eq. (4) and computing the roots of its derivative, one obtains the relation that the statistical uncertainty is minimal for an observation time that is exactly twice as long as the lifetime $\tau$ of the nuclide or about 2.9 times its half-life $T_{1 / 2}$. In practice, however, the observation time is limited either (i) for relatively long-lived ions, by residual-gas collisions that wash out the resonance beyond excitations times of about $10 \mathrm{~s}$, or (ii) for nuclides produced in very small numbers of less than about 100 ions/s by the restriction that at least a few hundred ions must be counted within some hours because otherwise an uncertainty contribution due to magnetic-field fluctuations become too large (this and other systematic-uncertainty contributions will be discussed in more detail in Sec. 5).

Equation (4) and the previous comments show the importance of the efficiency of the apparatus on the statistical uncertainty of the final result: The square root of the total number of recorded ions enters directly into the expression for the uncertainty. Furthermore, optimal observation times of twice the lifetime of a nuclide can only be reached if the efficiency is large enough to allow a few hundred ions to be recorded within a few hours. This means that for exotic nuclides that are produced at only a few hundred ions/s, the overall efficiency must be at least of the order of $10^{-3}$.

\section{$4 \quad$ RFQ ion beam cooler and buncher}

Up to 1998, a large hyperboloidal Paul trap was used as ISOLTRAP's beam conditioning trap $[19,20]$. In comparison with the rotating-foil scheme of the original setup [8], which relied on thermally induced reionization to liberate the deposited radioactive atoms, this Paul trap had the important merit of operating in a completely element-independent manner. It quickly became apparent, though, that its acceptance was not optimally matched to the phase space and energy dispersion of the 60-keV ISOLDE beam.

Linear radiofrequency quadrupole ion guides have been widely used as mass filters in chemistry [21, 22]. Their operating principle is based on the radial confinement of ions in the quadrupolar field of a four-rod structure, which can be described in terms of the Mathieu equations and their stability conditions [23, 24]. The time-averaged radial centering force can be expressed as a harmonic pseudo-potential well [25]. If the ion guide is filled with a light buffer gas, ions are not only radially confined but also cooled $[26,27$, 28]. An axial DC potential can be applied to the segmented rods in order to allow the accumulation of a number of ions in cooled bunches.

Such a linear radiofrequency ion trap system was designed [28] and implemented as ISOLTRAP's beam conditioning trap [29]. A sketch of the trap system is shown in Fig. 3. The $60-\mathrm{keV}$ ISOLDE ions are decelerated in a combination of deceleration and focusing electrodes and injected into the ion guide at a few tens of eV energy. In the buffer-gas-filled ion guide, whose total length is about $1 \mathrm{~m}$, the ions are radially confined and both radially and longitudinally cooled until they accumulate in a trap region near the end of the ion guide. Typically, the trap is operated at gas pressures of about $1 \mathrm{~Pa}$, at a radiofrequency of about $1 \mathrm{MHz}$, and at $\mathrm{RF}$ amplitudes of up to $250 \mathrm{~V}$ peak-to-peak, depending on the ion mass. After an accumulation and cooling time of a few ms, the cooled ion bunch is ejected towards the cooling Penning trap through a pulsed tube in which its potential energy is adapted to ground potential.

The performance of the newly installed RFQ ion beam cooler and buncher has been extensively studied [29]. The result of a measurement of the cooling time is shown in Fig. 4. In Fig. 4(a), the temporal width of the ejected ion pulse, recorded with an MCP detector located about $1 \mathrm{~m}$ downstream of the linear RFQ trap, is plotted as a function of 


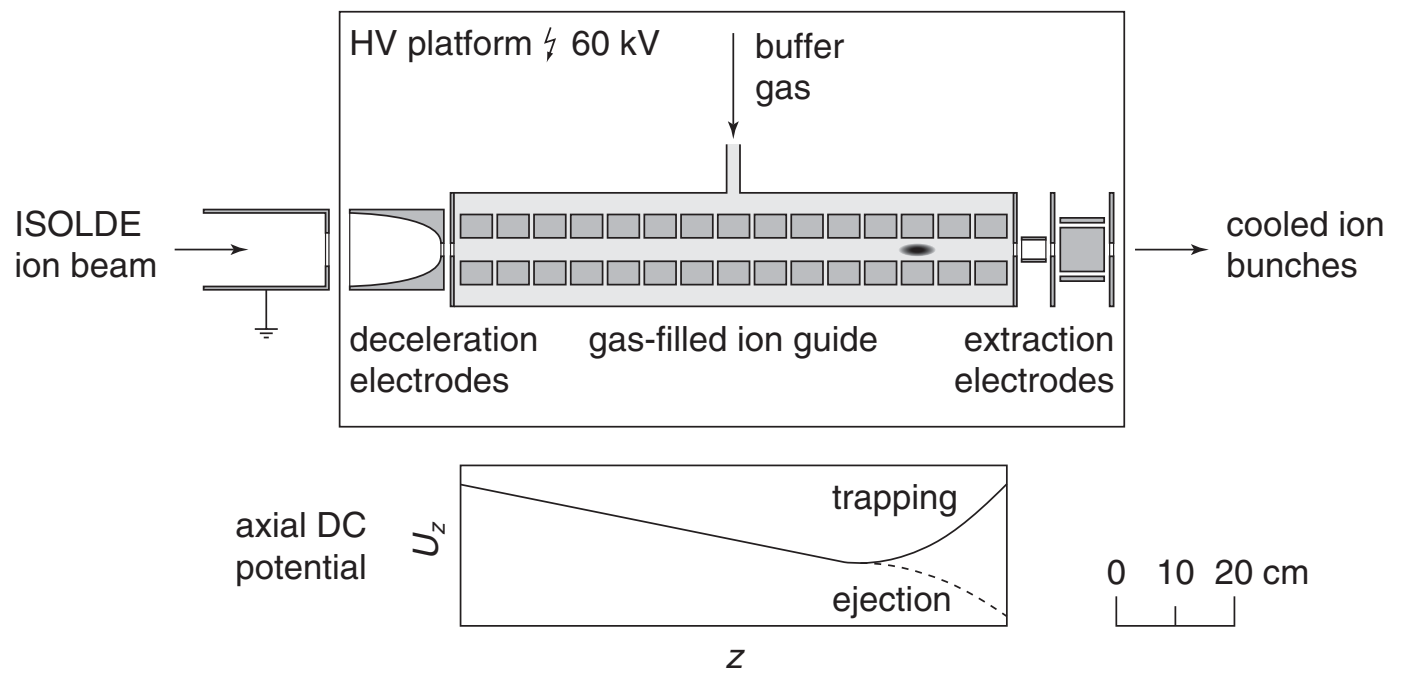

Figure 3: Sketch of the radiofrequency ion beam cooler and buncher. See the detailed description in the text.
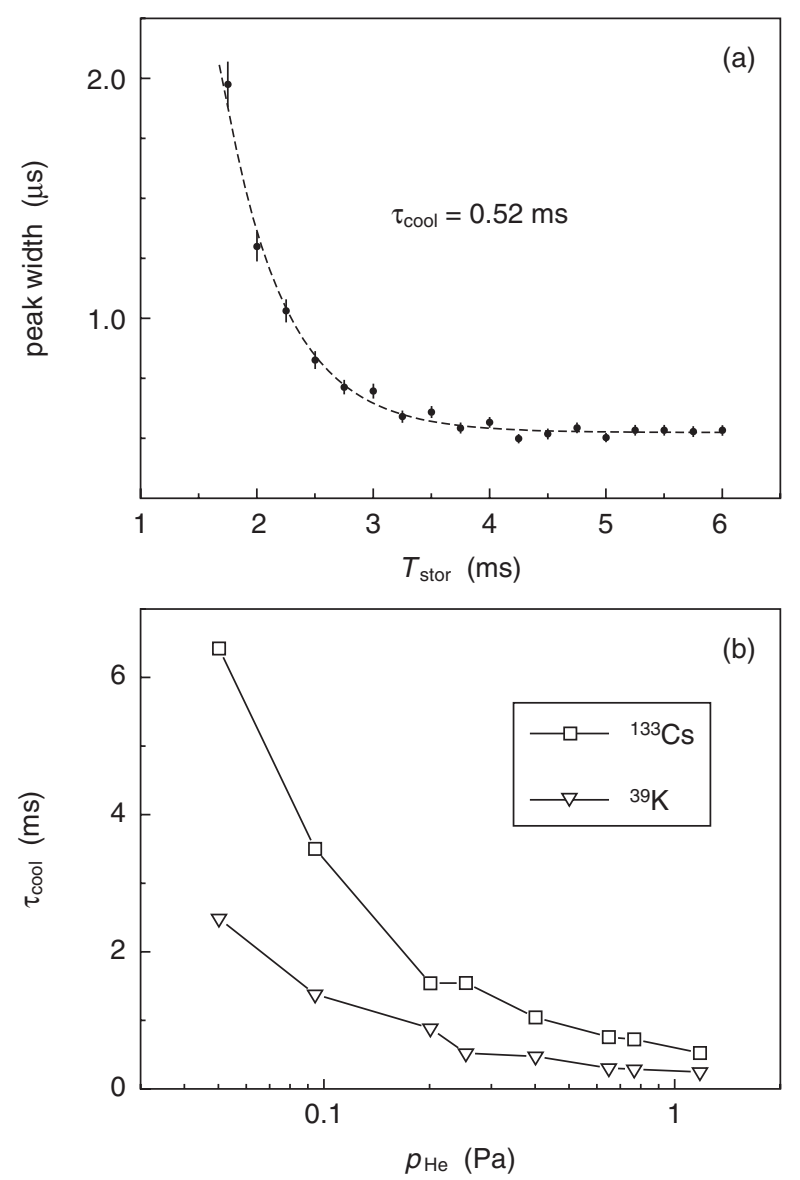

Figure 4: (a) Temporal width (FWHM) of the ejected ion cloud as a function of the storage time $T_{\text {stor }}$ in the linear RFQ ion trap, measured on an MCP detector about $1 \mathrm{~m}$ downstream [29]; (b) Dependence of the cooling time constant $\tau_{\text {cool }}$ on ion mass and buffer gas pressure [29]. The solid lines are meant to guide the eye. 
the storage time $T_{\text {stor }}$ in the trap. The data points were obtained with ${ }^{39} \mathrm{~K}$ ions at a helium buffer gas pressure of $0.25 \mathrm{~Pa}$. The solid line is an exponential fit to the data points. Its time constant $\tau_{\text {cool }}=0.52 \mathrm{~ms}$ can to first order be regarded as the cooling time constant of the ion cloud in the trap. After a storage time of a few $\tau_{\text {cool }}$, cooled ion pulses can be extracted from the trap.

Figure 4(b) shows the dependence of the cooling time constant $\tau_{\text {cool }}$ on the ion mass and the buffer gas pressure. With increased helium pressure, the cooling time constant is asymptotically reduced down to a few hundred $\mu \mathrm{s}$; an increase beyond about $1 \mathrm{~Pa}$ does not yield a further improvement. As would be expected, heavier ions require a longer storage time before they reach thermal equilibrium with the buffer gas.

Another important parameter of the beam conditioning trap is the final temperature reached by the ions. This property of the ion cloud can also be obtained from the temporal pulse width of an ion bunch after extraction from the trap. For this purpose, a measured pulse obtained with ${ }^{133} \mathrm{Cs}$ ions that were extracted from the RFQ ion beam cooler and buncher and accelerated to $30 \mathrm{keV}$ was compared to Monte-Carlo simulations based on initial ion distributions at various temperatures [29]. This comparison is shown in Fig. 5(a). The figure suggests that the ion cloud temperature which matches the measured pulse shape best is that for $300 \mathrm{~K}$, and it can be concluded from that agreement that after sufficient storage time, the ions are cooled to the temperature of the buffer gas, i.e. room temperature. The graph in Fig. 5(b) shows the calculated energy spectrum of the ion pulse for an initial ion cloud distribution as that given by the solid line in Fig. 5(a). Its energy dispersion is $\Delta E=3.7 \mathrm{eV}$. Combining these two results yields a longitudinal emittance of the extracted ion pulse of $\epsilon_{\text {long }}=10 \mathrm{eV} \mu \mathrm{s}$.

The transverse emittance of the ejected ion bunches at $2.5 \mathrm{keV}$ energy was measured with a beam observation system consisting of an MCP detector with a fluorescent screen and a beam scraper [29]. The beam observation setup was mounted about $2 \mathrm{~m}$ downstream of the RFQ trap, with the scraper $500 \mathrm{~mm}$ in front of the MCP detector. The radial beam extensions at the scraper and the MCP position were found to be $2.5 \mathrm{~mm}$ and $1.8 \mathrm{~mm}$, respectively (90 percent of the beam intensity within these radii) yielding a beam divergence of $5 \mathrm{mrad}$ and an upper limit of the transverse beam emittance of $\epsilon_{\text {trans }}=10 \pi \mathrm{mm}$ mrad. This corresponds to an emittance of about $2 \pi \mathrm{mm}$ mrad at $60 \mathrm{keV}$, a more than tenfold improvement over the incoming ISOLDE beam with a transverse emittance of about $35 \pi \mathrm{mm}$ mrad.

Most importantly, the RFQ ion beam cooler and buncher has proved to be significantly more efficient than the previously used system. The overall transmission in bunching mode was measured with various xenon isotopes delivered by ISOLDE as 30$\mathrm{keV}$ beams and found to be 12-15\% [29]. This constitutes an improvement by more than three orders of magnitude with respect to the large Paul trap setup. It is also safe to assume that the improved properties of the ion bunch extracted from the RFQ ion beam cooler and buncher have contributed to an additional increase of the transport efficiency between the traps as well as an enhanced capture efficiency in the cooling Penning trap.

\section{Carbon clusters for ISOLTRAP}

As mentioned above, the combined relative uncertainty of an ISOLTRAP mass measurement due to all systematic effects was estimated to be $1 \cdot 10^{-7}[8]$. In order to reach overall uncertainties at the level of $10^{-8}$, the individual uncertainty contributions had to be studied in more detail. Because carbon clusters have precisely known mass ratios ${ }^{1)}$, they 

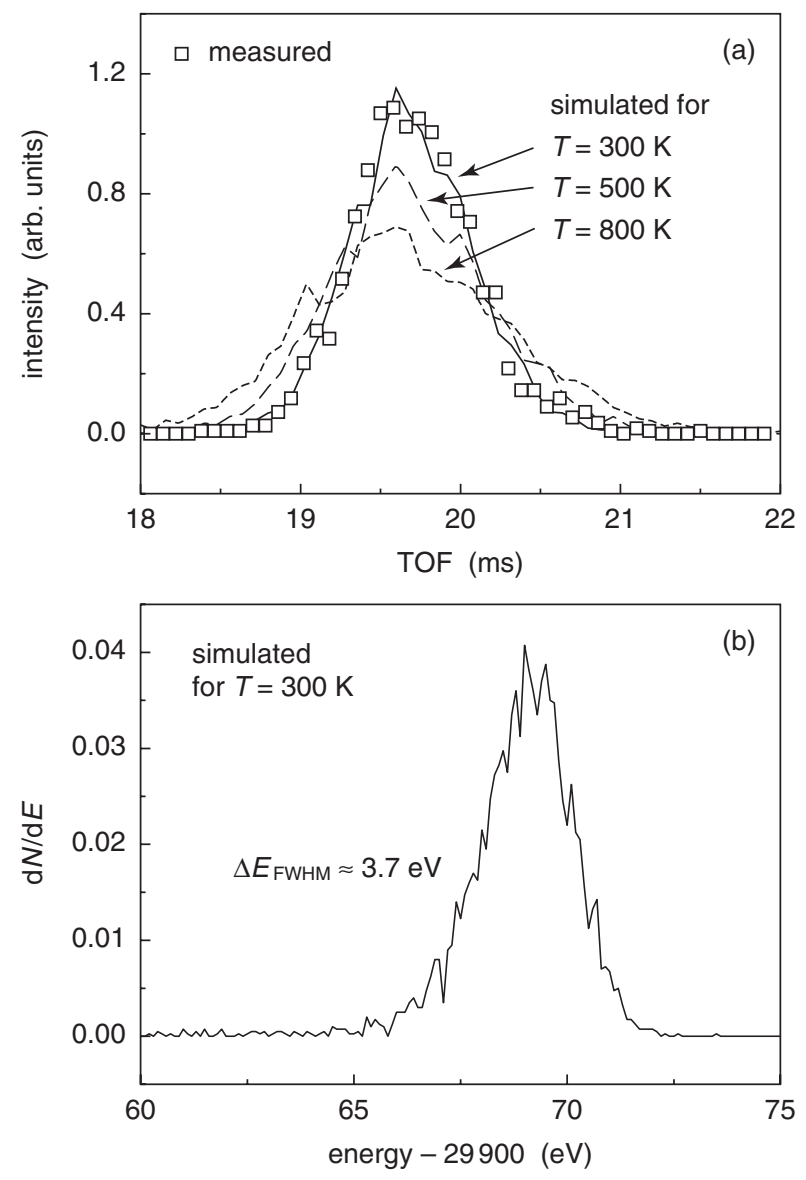

Figure 5: (a) Simulated temporal distribution of an ion pulse extracted from the RFQ ion beam cooler and buncher and accelerated to $30 \mathrm{keV}$ for different initial temperatures (lines) and measured distribution (squares) [29]; (b) Calculated energy spectrum of an ion pulse corresponding to the 300-K pulse shape of (a) [29]. All simulations and the measurement were performed with ${ }^{133}$ Cs.

represented an ideal test bed for a study of the accuracy of the ISOLTRAP apparatus. Aside from the small uncertainty associated with the molecular binding energies, the masses of clusters of ${ }^{12} \mathrm{C}$ are exactly known because the unified atomic mass unit $\mathrm{u}$ is defined in terms of that carbon isotope [32]. This means that if carbon clusters are used as reference ions for actual mass measurements, the uncertainty of the final mass result due to the uncertainty of the mass of the reference ion is virtually eliminated. Such measurements can be called absolute mass measurements because they relate the mass of an ion of interest directly to the microscopic mass standard.

Carbon cluster ions in a wide mass range have been produced from $\mathrm{C}_{60}$ fullerenes through laser-induced desorption, fragmentation, and ionization with ISOLTRAP's carbon cluster ion source [33]. A detailed sketch of this source, which was installed upstream of the cooling Penning trap in 1999, is shown in Fig. 6. The frequency-doubled beam of

1) According to theoretical calculations [30, 31], the molecular binding energies of carbon clusters follow a smooth behavior with magnitudes between 5.6 and $7 \mathrm{eV}$ per atom for $\mathrm{C}_{n}$ with $6 \leq n \leq 20$. The relative deviations of the mass ratios between the clusters from their rational-fraction relations are therefore always smaller than $10^{-9}$. 


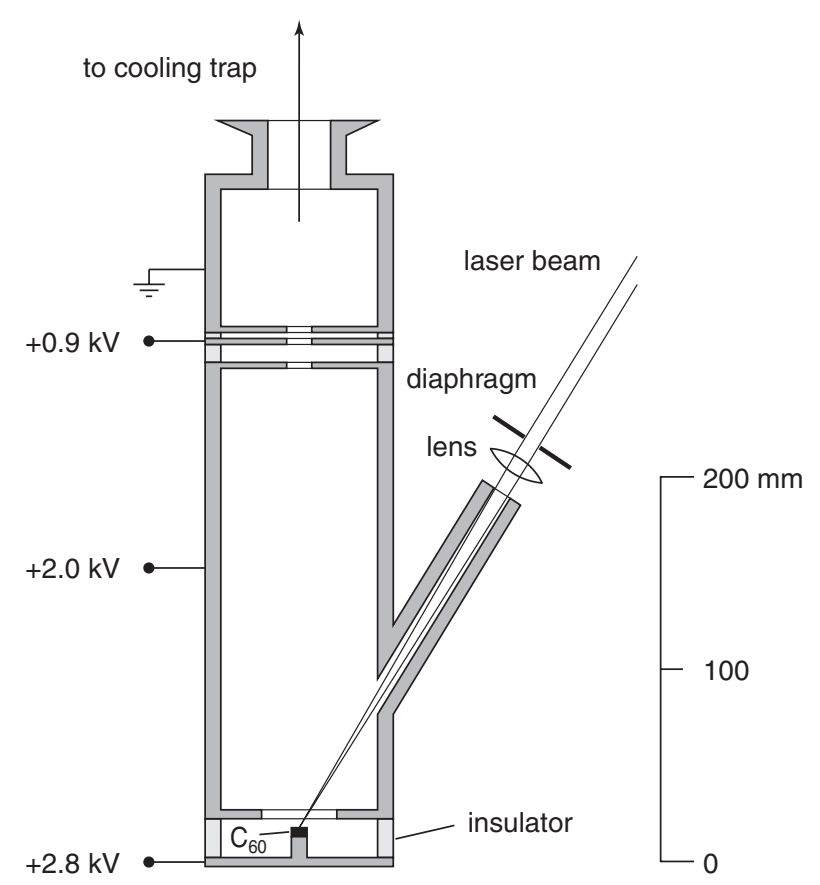

Figure 6: Detailed sketch of ISOLTRAP's carbon cluster ion source, which is mounted about $2.5 \mathrm{~m}$ upstream of the cooling Penning trap [33]. The carbon cluster ions are obtained from laser desorption, fragmentation, and photo-ionization of a $\mathrm{C}_{60}$ pellet and are electrostatically accelerated towards the cooling Penning trap.

a Q-switched Nd:YAG laser is focused on a $\mathrm{C}_{60}$ pellet; the desorbed and ionized fullerene fragments are electrostatically accelerated to the transfer potential of about $2.8 \mathrm{keV}$ and transferred to the cooling Penning trap.

A TOF mass spectrum recorded with an MCP detector about $1 \mathrm{~m}$ downstream of the carbon cluster ion source is shown in Fig. 7(a) [34]. The cluster ions thus produced were mass-selectively cooled in the cooling Penning trap in order to isolate a single cluster size $\mathrm{C}_{n}$. The desired clusters were then transferred to the precision Penning trap for standard cyclotron-frequency measurements. Neither the buffer gas cooling nor the RF excitations in the two traps led to any significant losses due to fragmentation or charge exchange. Figure 7(b) depicts a typical TOF resonance obtained in the precision Penning trap with $\mathrm{C}_{9}^{+}$ions at an excitation duration of $T_{\mathrm{Q}}=1.5 \mathrm{~s}[18]$.

For a detailed study of the systematic effects that contribute to the uncertainty of a mass measurement, beyond the well-understood effects due to the presence of contaminant ions in the precision Penning trap $[17,35]$ and due to magnetic-field fluctuations between the two reference measurements [12], 114 so-called cross-reference measurements in a mass range from $72 \mathrm{u}$ to $240 \mathrm{u}$ (clusters $\mathrm{C}_{6}$ to $\mathrm{C}_{20}$ ) were carried out [18]. These are measurements in which the cyclotron frequencies of two carbon cluster ions with different mass are compared. A deviation of the measured mass ratios from their true values which was proportional to the mass difference between any pair of clusters was found and interpreted as a mass-dependent systematic error. Every single mass ratio measurement is therefore subject to an uncertainty whose magnitude is proportional to the mass difference between the ion of interest and the reference ion:

$$
\frac{u_{\mathrm{m}}(m)}{m}=1.6(4) \cdot 10^{-10} / \mathrm{u} \cdot\left(m-m_{\mathrm{ref}}\right) .
$$



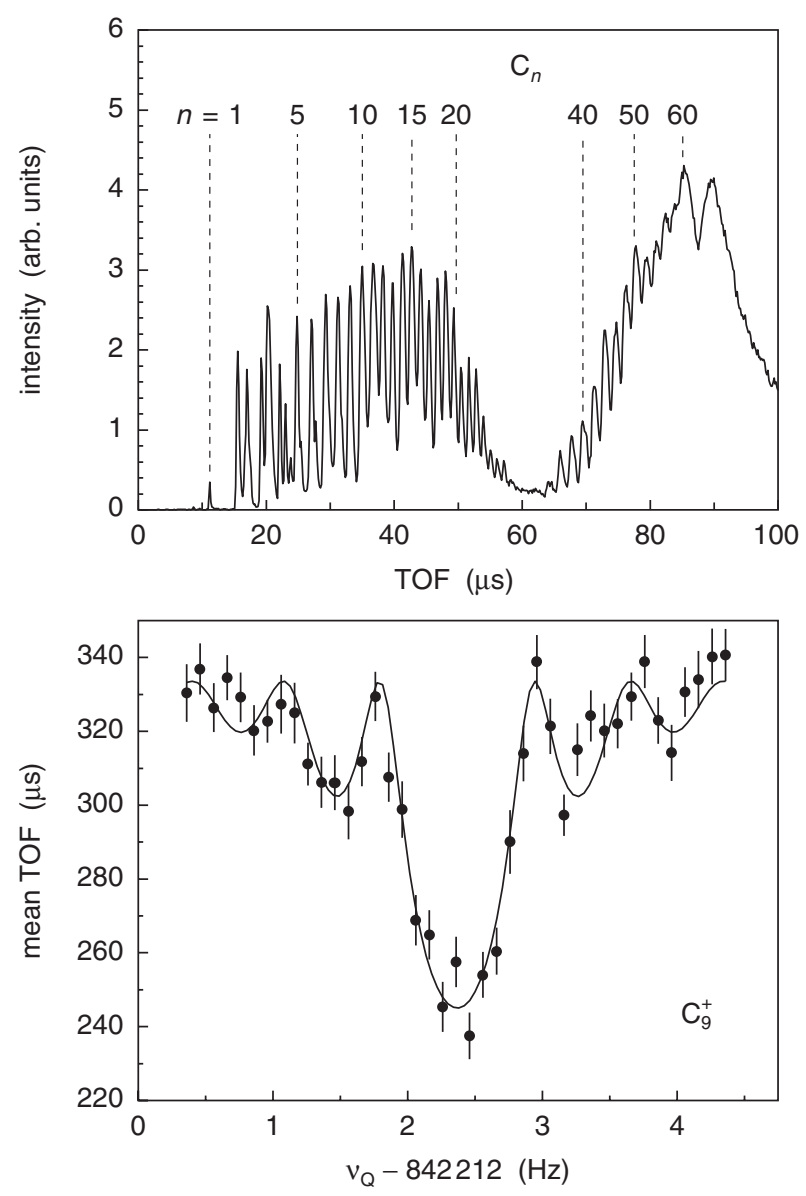

Figure 7: (a) Time-of-flight mass spectrum of carbon cluster ions produced with ISOLTRAP's carbon cluster ion source, recorded on an MCP detector mounted about $1 \mathrm{~m}$ above the source [34]; (b) Time-of-flight resonance of $\mathrm{C}_{9}^{+}$[18]. The solid line is a fit fit of the expected line shape [17] to the data points.

Such a mass-dependent deviation can be due to imperfections of the electric-quadrupole field or a misalignment of the magnetic field with respect to the electrostatic trapping fields.

With a knowledge of the magnitude of the mass-dependent effect, the corrected weighted mean masses for all cross-reference measurements were computed and a $\chi^{2}$ test performed in order to determine the residual systematic uncertainty of the apparatus [18]. A plot of the deviations of these weighted mean masses from their true values is shown in Fig. 8. The reduced $\chi^{2}$ of these 11 data points that represent a total of more than 300 TOF resonance measurements was found to be $\chi^{2} / N \approx 2$, where $N=11$ is the number of averaged mass results. In order for this reduced $\chi^{2}$ to be lowered to one, it was necessary to quadratically add to each mean value a relative uncertainty of

$$
\frac{u_{\mathrm{res}}(m)}{m}=8 \cdot 10^{-9}
$$

which thus represents the accuracy limit of the ISOLTRAP apparatus. 


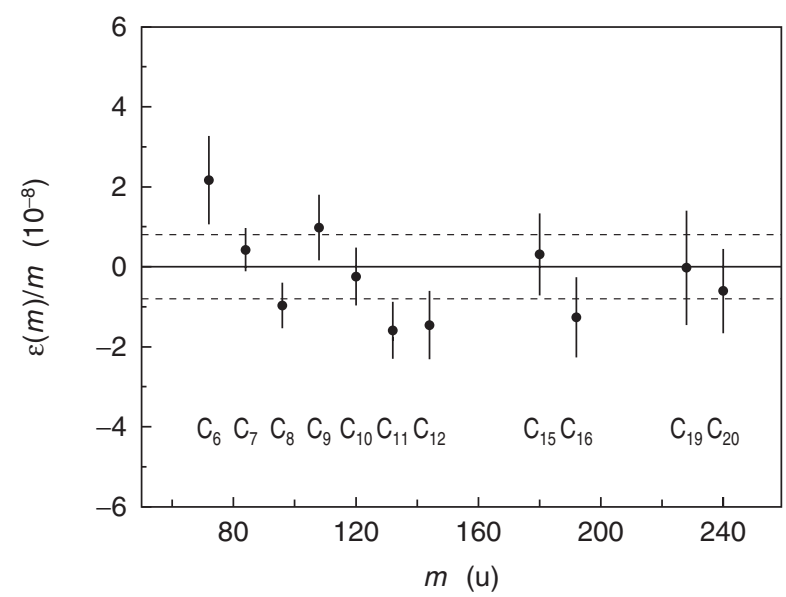

Figure 8: Deviations of the weighted means of the measured carbon cluster ion masses from their true values after correction for the mass-dependent systematic effect [18]. The dashed lines indicate the residual systematic uncertainty.

\section{Phase locking of the magnetron excitation}

As opposed to the idealized model according to which ions initially have no radial energy in the precision Penning trap, a slight magnetron motion with a radius of a few tenths of millimeters cannot be avoided. While the radius of this motion is small compared to that which the ions acquire through the dipolar excitation, its phase destroys the arbitrariness of the phase of the subsequent excitations.

The phase difference between the dipolar excitation and the initial magnetron motion, $\Delta \phi_{-}=\phi_{\mathrm{D}}-\phi_{-}(0)$, has quantifiable repercussions on the shape of the observed TOF resonance [36]: If the phase of the dipolar excitation precedes that of the initial motion by $\pi / 2$, the irradiated energy adds directly to the magnetron motion's energy. If the phase difference is $3 \pi / 2$, the magnetron radius is first reduced to zero and then increased again, but to a smaller final magnitude than for $\Delta \phi_{-}=\pi / 2$. This effect is shown in detail in Fig. 9(a), where the calculated final magnetron radius $\rho_{-}$of the ions is shown as a function of the dipolar-excitation duration $T_{\mathrm{D}}$. The figure also shows that for intermediate phases, the magnetron radius increases slowly at first, then almost linearly. In Fig. 9(b), the calculated final magnetron radius $\rho_{-}$is plotted against the phase difference $\Delta \phi_{-}$for different values of the dipolar-excitation durations $T_{\mathrm{D}}$. For rather long excitation durations, such as those employed at ISOLTRAP, the final magnetron radius is never zero, but a non-optimal choice of the phase can reduce its magnitude significantly.

This expected effect was confirmed with a measurement using ${ }^{39} \mathrm{~K}$ ions from the reference ion source [36]. The experimental data shown in Fig. 10 confirms the calculations very nicely. This curve should be compared to the dotted curve in Fig. 9(b); the deviation from the sinusoidal shape is due to the non-linearity of the conversion of radial modifiedcyclotron motion to linear motion in the inhomogeneous-magnetic-field region.

Since the modified-cyclotron radius obtained after the resonant quadrupolar excitation can at most be as large as the magnetron radius that the ions had prior to the conversion, this reduction of the magnetron radius directly translates into a reduced gain in energy in the inhomogeneous-field region and thus into a time of flight that is not as strongly reduced as with a more optimal phase difference. This effect is shown in Fig. 11, which shows recorded TOF resonances for the three delays between the start of the mea- 

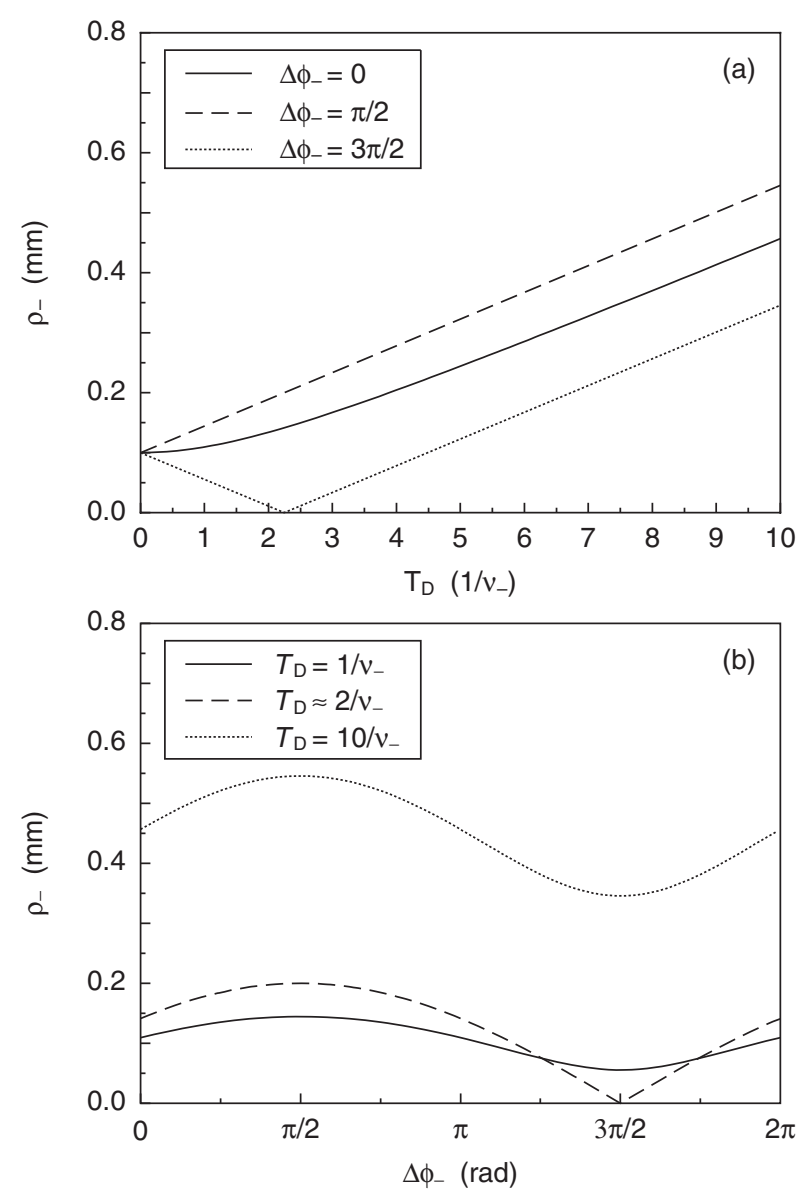

Figure 9: (a) Calculated final magnetron radius as a function of the duration of the dipolar excitation for three values of the phase difference between the excitation and the initial magnetron motion [36]; (b) Calculated final magnetron radius as a function of the phase difference between the excitation and the initial magnetron motion for three different excitation durations [36].

surement cycle and that of the magnetron excitation marked by arrows in Fig. 10 [36]. The three graphs show the progression from the least optimal phase relation to the ideal one. The relative difference in time of flight between the baseline and the centroid minimum, which is also called the TOF effect, increases strongly between graphs (1) and (3). Since the statistical uncertainty of the fit to the data points depends mostly on the data points in the flanks of the centroid, it is expected to behave inversely. This is confirmed by the experimentally obtained TOF resonances, which all contain similar numbers of ions $N_{\text {tot }}$ : while the TOF effect increases from $14.4 \%$ in graph (1) to $41.9 \%$ in graph (3), the relative statistical uncertainty $s\left(\nu_{\mathrm{c}}\right) / \nu_{\mathrm{c}}$ decreases from $5.8 \cdot 10^{-8}$ to $1.3 \cdot 10^{-8}$.

These results indicate that the precision of the measurement can be significantly increased by fixing the phase difference between the initial ion magnetron motion in the precision Penning trap and the dipolar magnetron excitation to $\Delta \phi_{-}=\pi / 2$. The actual gain in precision obtained by this modification is given by the ratio of the statistical uncertainty with $\Delta \phi_{-}=\pi / 2$ to that with an arbitrary phase, equivalent to $\Delta \phi_{-} \approx 0$. It was found from the measurements discussed above that the reduction in statistical uncertainty is about $1 / 3$, an effect which would be equivalent to more than doubling the 


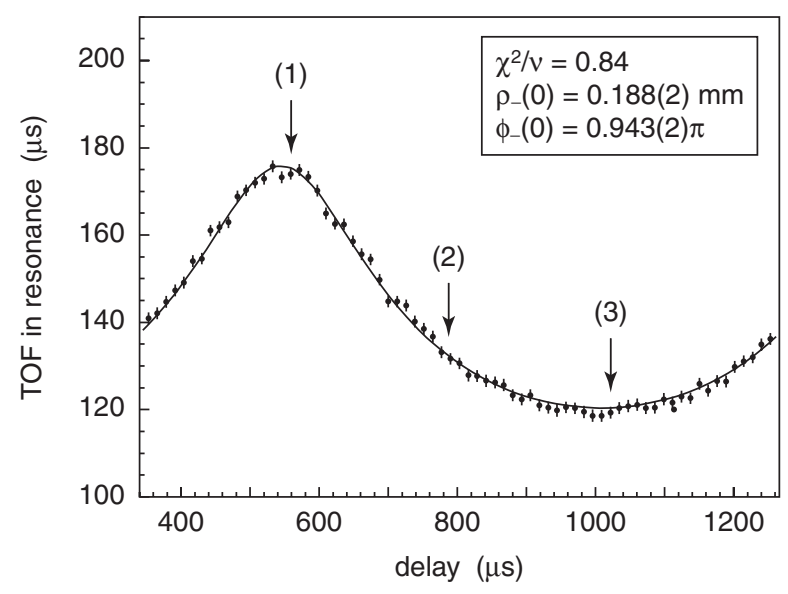

Figure 10: Time of flight in resonance, i.e. at the centroid minimum, as a function of the delay between the start of the measurement cycle and that of the magnetron excitation, corresponding to a phase difference of 0 to $2 \pi$ [36]. The delays marked by arrows correspond to the phase differences (1) $\Delta \phi_{-}=3 \pi / 2$, (2) $\Delta \phi_{-} \approx 0$, and (3) $\Delta \phi_{-}=\pi / 2$. The solid line is a fit of the theoretical curve to the data points.

beam intensity.

\section{$7 \quad$ Conclusion}

The installation of the new beam preparation trap, a linear RFQ quadrupole ion beam cooler and buncher, as well as some minor mechanical improvements not discussed here, have led to a combined increase of the overall efficiency of the apparatus of more than three orders of magnitude. Without considering losses due to radioactive decay, the total transmission through all three traps is now of the order of $1 \%$. The implementation of a phase locking mechanism for the dipolar magnetron excitation in the precision trap has made possible a further improvement of the statistical uncertainty by a factor $1 / 3$, which brings the total increase in statistical measurement precision for the developments described here to almost a factor 100 with respect to the status of 1998. In the cases where the final uncertainty is limited by the residual systematic uncertainty given in Eq. (6), the increased statistical precision translates to a strongly reduced measurement time, an important asset for an experiment at an on-line facility.

With the design and installation of a carbon cluster ion source, it was possible to demonstrate the mass-selective cooling and cyclotron frequency measurements of carbon cluster ions in a wide mass range. The various clusters were produced in sufficient quantities to allow a detailed study of the systematic uncertainties that affect mass measurements. This study revealed that the relative accuracy limit of the apparatus is $8 \cdot 10^{-9}$, more than an order of magnitude smaller than previously estimated. The carbon cluster source can of course also be used as a reference ion source, thus expanding the mass range of available reference ions and at the same time making available reference ions whose mass difference from the ion of interest is at most $6 \mathrm{u}$. In the near future, it will be possible to perform absolute mass measurements with ISOLTRAP, i.e. mass measurements relative to the microscopic mass standard.

The combination of these advances has for the first time allowed mass measurements on radioactive nuclides with half-lives well below $100 \mathrm{~ms}$ and with an overall relative mass 


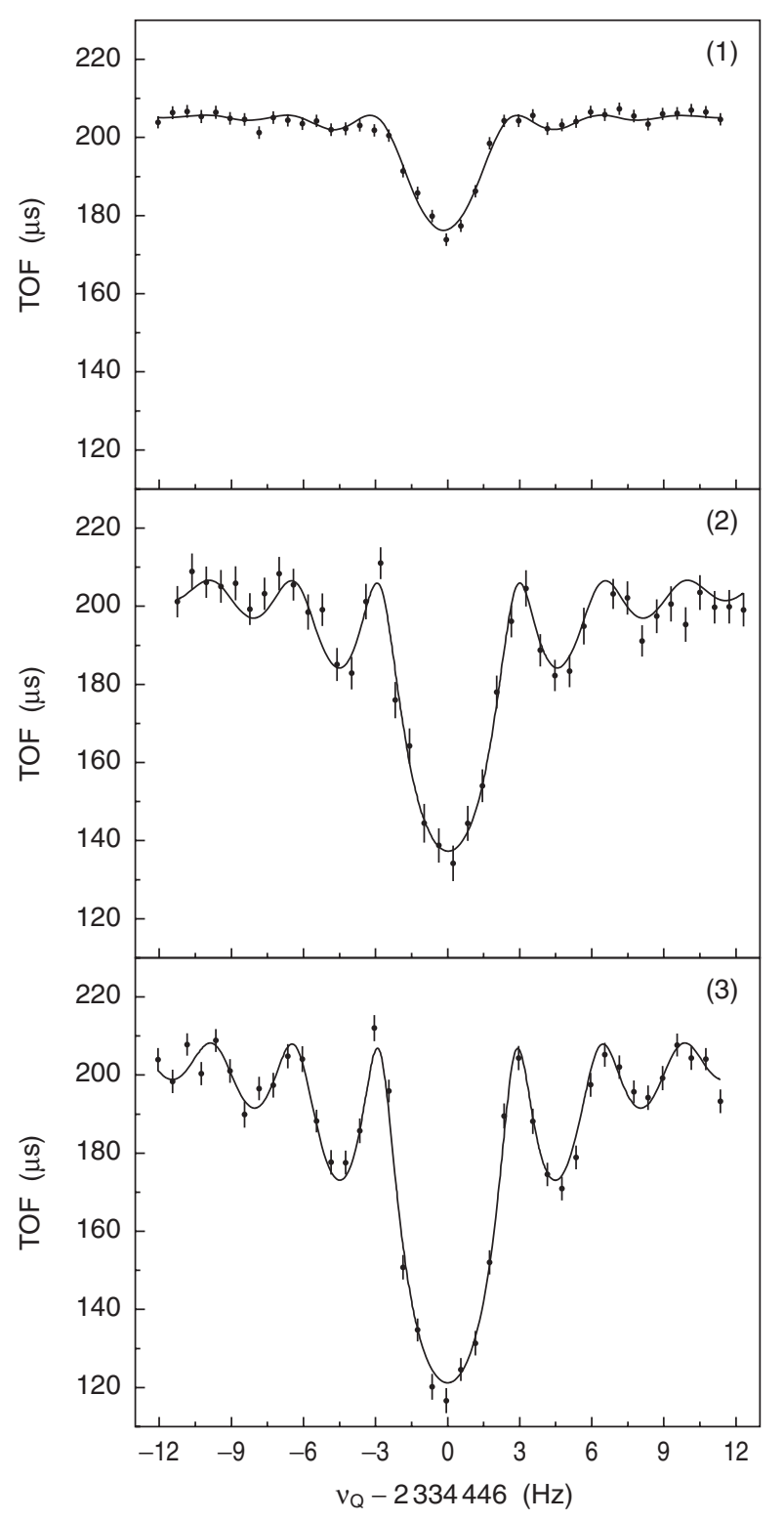

Figure 11: Time-of-flight resonances recorded with delays between the start of the measurement cycle and that of the magnetron excitation corresponding to the three phases marked by arrows in Fig. 10 [36]. The solid line are fits of the theoretical function [17] to the data points.

uncertainty of about $10^{-8}[37,38,39]$, thereby reaffirming ISOLTRAP's role as one of the leading experiments in high-precision mass spectrometry of exotic nuclides.

The carbon cluster tests have also shown that the technique of TOF cyclotron resonance detection is well suited for mass measurements on large molecules. With the clusters studied here, the obtained resolving powers range from about 570000 for $\mathrm{C}_{20}$ to about 2000000 for $\mathrm{C}_{6}$ with an excitation duration of $1.5 \mathrm{~s}$. If, for the sake of comparison, this result is extrapolated to mass number 1000 and an excitation time of $10 \mathrm{~s}$, a resolving power of almost $10^{6}$ is found. Owing to the fact that charge states higher than one are not preserved in the cooling Penning trap and that residual-gas collisions limit the observation time to about $10 \mathrm{~s}$, this falls short by about a factor 4 of the highest molecular mass 
resolving power in this mass range reported so far [40], obtained with the Penning trap at Florida State University using the Fourier transform ion cyclotron resonance (FT-ICR) technique. However, since single-ion detection does not depend on simultaneous baseline resolution of two ion species, ISOLTRAP would allow a resolved mass difference of about $10^{-5} \mathrm{u}$ in that mass range.

According to the calculations of carbon cluster binding energies mentioned above, the ratio of the total molecular binding energy to the mass is about $6 \cdot 10^{-10}$. It therefore follows from Eq. (6) that the overall precision of ISOLTRAP would have to be improved by about two orders of magnitude in order to be able to carry out direct measurements of the molecular binding energy. This could be achieved by a combination of both higher charge states and longer observation times, the latter of which would be made possible by an improved vacuum in a precision trap that is cooled to liquid-nitrogen temperature.

\section{References}

[1] D. Lunney, G. Bollen, Hyp. Int. 129 (2000) 249.

[2] S. Schwarz, F. Ames, G. Audi, D. Beck, G. Bollen, C. De Coster, J. Dilling, O. Engels, R. J. E. García-Ramos, S. Henry, F. Herfurth, K. Heyde, A. Kellerbauer, H.-J. Kluge, A. Kohl, E. Lamour, M. D. Lunney, I. Martel, R. B. Moore, M. Oinonen, H. Raimbault-Hartmann, C. Scheidenberger, G. Sikler, J. Szerypo, C. Weber, Nucl. Phys. A 693 (2001) 533.

[3] M. Arnould, K. Takahashi, Rep. Prog. Phys. 62 (1999) 395.

[4] J. C. Hardy, I. S. Towner, Hyp. Int. 132 (2001) 115.

[5] T. Fritioff, C. Carlberg, G. Douysset, R. Schuch, I. Bergström, Eur. Phys. J. D 15 (2001) 141.

[6] S. Brunner, T. Engel, A. Schmitt, G. Werth, Eur. Phys. J. D 15 (2001) 181.

[7] S. Rainville, J. K. Thompson, D. E. Pritchard, Can. J. Phys. 80 (2002) 1329.

[8] G. Bollen, S. Becker, H.-J. Kluge, M. König, R. B. Moore, T. Otto, H. RaimbaultHartmann, G. Savard, L. Schweikhard, H. Stolzenberg, the ISOLDE Collaboration, Nucl. Instrum. Methods A 368 (1996) 675.

[9] E. Kugler, Hyp. Int. 129 (2000) 23.

[10] G. Bollen, Nucl. Phys. A 693 (2001) 3.

[11] F. Herfurth, F. Ames, G. Audi, D. Beck, K. Blaum, G. Bollen, A. Kellerbauer, H.-J. Kluge, M. Kuckein, D. Lunney, R. B. Moore, M. Oinonen, D. Rodríguez, E. Sauvan, C. Scheidenberger, S. Schwarz, G. Sikler, Weber, the ISOLDE Collaboration, J. Phys. B 36 (2003) 931.

[12] D. Beck, F. Ames, G. Audi, G. Bollen, H.-J. Kluge, A. Kohl, M. König, D. Lunney, H. Raimbault-Hartmann, S. Schwarz, J. Szerypo, Nucl. Instrum. Methods B 126 (1997) 374.

[13] M. P. Bradley, J. V. Porto, S. Rainville, J. K. Thompson, D. E. Pritchard, Phys. Rev. Lett. 83 (1999) 4510.

[14] H. Raimbault-Hartmann, D. Beck, G. Bollen, M. König, H.-J. Kluge, E. Schark, J. Stein, S. Schwarz, J. Szerypo, Nucl. Instrum. Methods B 126 (1997) 378.

[15] G. Savard, S. Becker, G. Bollen, H.-J. Kluge, R. B. Moore, T. Otto, L. Schweikhard, H. Stolzenberg, U. Wiess, Phys. Lett. A 158 (1991) 247.

[16] L. S. Brown, G. Gabrielse, Rev. Mod. Phys. 58 (1986) 233.

[17] M. König, G. Bollen, H.-J. Kluge, T. Otto, J. Szerypo, Int. J. Mass Spectrom. Ion Proc. 142 (1995) 95. 
[18] A. Kellerbauer, K. Blaum, G. Bollen, F. Herfurth, H.-J. Kluge, M. Kuckein, E. Sauvan, Eur. Phys. J. D 22 (2003) 53.

[19] R. B. Moore, G. Rouleau, J. Mod. Optics 39 (1992) 361.

[20] S. Schwarz, Ph.D. thesis, University of Mainz (1999).

[21] E. Teloy, D. Gerlich, Chem. Phys. 4 (1974) 417.

[22] D. Gerlich, Adv. Chem. Phys. 82 (1992) 1.

[23] P. H. Dawson, Quadrupole Mass Spectrometry, Elsevier, Amsterdam, 1976.

[24] W. Paul, Rev. Mod. Phys. 62 (1990) 531.

[25] H. G. Dehmelt, Adv. At. Mol. Phys. 3 (1967) 53.

[26] A. F. Tolmachev, A. V. Chernushevich, I. V. Dodonov, K. G. Standing, Nucl. Instrum. Methods B 124 (1997) 112.

[27] D. Lunney, R. B. Moore, Int. J. Mass Spectrom. Ion Proc. 190/191 (1999) 153.

[28] A. Kellerbauer, T. Kim, R. B. Moore, P. Varfalvy, Nucl. Instrum. Methods A 469 (2001) 276.

[29] F. Herfurth, J. Dilling, A. Kellerbauer, G. Bollen, S. Henry, H.-J. Kluge, E. Lamour, D. Lunney, R. B. Moore, C. Scheidenberger, S. Schwarz, G. Sikler, J. Szerypo, Nucl. Instrum. Methods A 469 (2001) 254.

[30] B. R. Eggen, R. L. Johnston, J. N. Murrell, J. Chem. Soc. Farad. Trans. 90 (1994) 3029 .

[31] F. R. Manby, R. L. Johnston, C. Roberts, Commun. Math. Comp. Chem. 38 (2001) 111.

[32] T. J. Quinn, I. M. Mills, The International System of Units (SI), 7th Edition, Bureau International des Poids et Mesures, Sèvres (France), 1998.

[33] C. Scheidenberger, G. Bollen, F. Herfurth, A. Kellerbauer, H.-J. Kluge, M. Koizumi, S. Schwarz, L. Schweikhard, Nucl. Phys. A 701 (2002) 574c.

[34] K. Blaum, G. Bollen, F. Herfurth, A. Kellerbauer, H.-J. Kluge, M. Kuckein, E. Sauvan, C. Scheidenberger, L. Schweikhard, Eur. Phys. J. A 15 (2002) 245.

[35] G. Bollen, H.-J. Kluge, M. König, T. Otto, G. Savard, H. Stolzenberg, R. B. Moore, G. Rouleau, G. Audi, the ISOLDE Collaboration, Phys. Rev. C 46 (1992) R2140.

[36] K. Blaum, G. Bollen, F. Herfurth, A. Kellerbauer, H.-J. Kluge, S. Heinz, P. Schmidt, L. Schweikhard, J. Phys. B 36 (2003) 921.

[37] F. Herfurth, A. Kellerbauer, F. Ames, G. Audi, D. Beck, K. Blaum, G. Bollen, O. Engels, H.-J. Kluge, D. Lunney, R. B. Moore, M. Oinonen, E. Sauvan, C. Scheidenberger, S. Schwarz, G. Sikler, C. Weber, the ISOLDE Collaboration, Eur. Phys. J. A 15 (2002) 17.

[38] A. Kellerbauer, Ph.D. thesis, University of Heidelberg, <http://www.ub.uniheidelberg.de/archiv/2463> (2002).

[39] K. Blaum, G. Bollen, F. Herfurth, H.-J. Kluge, A. Kellerbauer, E. Sauvan, S. Schwarz, the ISOLDE Collaboration, Phys. Rev. Lett. (submitted).

[40] F. He, C. L. Hendrickson, A. G. Marshall, Anal. Chem. 73 (2001) 647. 